\title{
FONTANUS CUMULATIVE INDEX TO VOLUMES I TO X
}

This cumulative index is comprised of four indexes: Author, Title, Subject and Illustration Index.

References are to volume numbers and page(s). Page numbers in parentheses refer to unnumbered pages. The Subject Index is a permuted key-word index. Key words were derived from the titles, abstracts and general content of the articles. All illustrations have been included in the Illustration Index save for reproductions of pages from books and manuscripts.

Since the publication pattern of Fontanus has been irregular, volumes and corresponding years are the following:

$\begin{array}{rr}\text { Volume I (1988) } & \text { Volume VI (1993) } \\ \text { II (1989) } & \text { VII (1994) } \\ \text { III (1990) } & \text { VIII (1995) } \\ \text { IV (1991) } & \text { IX (1996) } \\ \text { V (1992) } & \text { X (1998) }\end{array}$

\section{FONTANUS AUTHOR INDEX}

Alston, Sandra V, 235

Anderson, Helen VIII, 147

Axelrod, Paul X, 17

Bayley, Susan I, 105

Bell, Allan VI, 45

Berdnikov, Alexander VIII, 147

Berg, David III, 117

Binnie, Ian VII, 33

Bisson, Michael VII, 53

Blackwell, Miles IX, 25

Boulianne, Réal G. V, 55

Boyle, Christina M. X, 65

Brendel, Maria L. VIII, 109;

$\mathrm{X}, 35$

Brierley, J.E.C. I, 77
Carle, Paul III, 77

Cartwright, Michael IX, 7

(Foreword)

Caya, Marcel VII, 149

Clarke, Robert F. X, 110

Cohen, Marilyn IV, 175

Cohen, Martin Vl, 143

Cohen, Montague I, 3; II, 111; IV, 69; V, 123; VII, 155 ,

183; X, 7 (Editorial), 11

Cyr, Mary III, 35

Davis, Davena II, 27

Dawson, Sir William VI, 7

Dolphin, Bruce IX, 59

Dubuc, Alfred I, 69
Dufresne, Madeleine III, 77

Dunbar, M.J. IV, 7

Edel, Leon III, 5

Entin, Martin A. VIII, 137

Frost, Stanley Brice III, 65; VI, 85; VII, 41; VIII, 59; IX, 9

Gacek, Adam II, 37; III, 45;

IV, $35 ; \mathrm{X}, 41$

Gagnon, Hervé X, 71

Gareau-Des Bois, Louise VII, 113

Gascon, France III, 151

German, Daniel III, 109

Gillett, Margaret. VIII, 13 
Graham, Conrad E.W. III, 151; IV, 179; VI, 162; VII, 164; IX, 115

Grew, John VI, 147

Groen Frances II, 55

Gunn, Kevin IX, 120

Hanlon, Peter VIII, 49

Harvie, Ron VII, 158

Hatzopoulos, Dionysios IV, $55 ; \mathrm{V}, 7$

Hobbins, A.J. I, 59; II, 7;

IV, 143; VI, 119; VIII, 121;

$\mathrm{X}, 112$

Johnston, David L. IX, 23

Kennell, Elizabeth H. V, 223

Klibansky, Raymond II, 65

Lawson, Barbara IV, 109;

VII, 53

Leacock, Stephen VII, 7

Lindeman, Yehudi V, 229;

VII, 103

Liscombe, Rhodri W. I, (49b)

Lomer, Gerhard R. VI, 16

Marley, Carol X, 116

McKinnon, Alastair V, 173
McKnight, David IX, 118

McNally, Peter F. I, 95; VI, 69; VII, 152, 181; IX, 120; X, 65,93

Michel, Robert V, 33; VI, 97, 149; VII, 73; VIII, 59; IX, 29, 125

Miller, Pamela J. III, 17

Mitchell, Sydney B. VI, 13

Möller, Hans I, 2 (Editorial);

II, 5 (Editorial); VIII, 7

(Editorial); 97; IX, 131; X, 9

Mongeau, Alain III, 77

Morgan, Norma V, 73

Murray, Irena Žantovská IV, 125,183

Nadeau, Christine D. X, 71

Ormsby, Eric IV, 5 (Dedication); VI, 5 (Introduction)

Pan, Jason VII, 174

Pappius, Hanna. M. VI, 156

Pummer, Reinhard V, 161

Reid-Walsh Jacqueline IV, 175

Renshawe, M.L. I, 77

Schlosser, Franziska E. IV, 109
Schoenauer, Norbert IX, 47

Shapiro,Elizabeth V, 217

Sigal, Goldie I, 84

Syrek, Barbara VII, 127

Teather, Lynne III, 77

Thomson, George X, 101

Toope, Stephen J. X, 81

Trigger, Bruce VII, 53

Troide, Lars I, (37b)

Turpin, Rosemary VIII, 27

Van Peteghem, Alfred VII, 170

Vogel, Robert III, 131

Virr, Richard I, (117b); II, 103; IV, 109; V, 7

Whitehead, M.A. IX, 105

Whiteman, Bruce II, 97; IV, 181; VII, 11

Williams, Kate X, 115

Woloch, G. Michael IV, 109

Wright, Donald A. IX, 97

Wysocki, Leszek I, (117b)

Yaycioğlu, Ali X, 41 


\section{FONTANUS TITLE INDEX}

Arabic Calligraphy and the "Herbal" of al-Ghâfiqî: A Survey of Arabic Manuscripts at McGill University II, 37

(The) Archaeological Context of the Egyptian and Nubian Antiquities in the Redpath Museum III, 117

(An) Archival Testament: The Papers of Sir William Dawson (Notes and Comments) IX, 125

(Les) Archives Photographiques Notman du Musée McCord VII, 127

(The) Austin "Dink" Carroll Papers in the University Archives (Notes and Comments) VI, 149

Beatrice V. Simon (1899-1994) (Notes and Comments) VII, 181

Books on Old Violins and 19th-Century Playing From the Bequest of T. Wesley Mills III, 35

Breaking the Silence and Bridging the Gap: Documenting Personal Memories of the Holocaust (Notes and Comments) V, 229

(A) Brief Survey of Science and Scientists at McGill IX, 105

British Parliamentary Papers as Sources for Modern Language Teaching Research I, 105

Canada's Animal Doctor: Duncan McNab McEachran X, 101

Canadian Unity and Quebec in 1942: A Roundtable Discussion Among John Humphrey, Hugh

MacLennan and Émile Vaillancourt VI, 119

Celebrating Peter Redpath and His Library VI, 7

Charles deVolpi - Collector Extraordinary (Notes and Comments) III, 153

(A) Collection of Qur'anic Codices IV, 35

(La) Contribution de Maude Abbott au développement de la muséologie médicale (1898-1940) X, 71

(The) Correspondence of The Royal Institution for the Advancement of Learning in the McGill

University Archives V, 55

Cultural Diversity and Human Rights X, 81

(A) Danish Collection at McGill IX, 131

Deluxe Devotional Prayer Books: A McGill Book of Hours VIII, 109

Diaries from the McGill University Archives - A Sampling V, 3

Dignified and Picturesque: Redpath Library in 1893 VI, 69

(Les) documents et artifacts à l'Université McGill I, 69

(The) Earliest Canadian Music Programme (Notes and Comments) V, 235

(An) Early Canadian Herbal (Notes and Comments) VII, 170

Early Children's Books in the McGill University Libraries (Notes and Comments) IV, 175

Early Qur'anic Fragments III, 45

Excavating Collections: Archaeological Finds at the Redpath Museum VII, 53

(The) F. R. Scott Library II, 97 
Floreat Plutoria: Satirical Fiction About McGill IX, 29

Frank Cyril James: The Principal Years III, 65

Frank Scott and the Canadian Literary Renaissance III, 5

(A) Frank Scott Letter from 1937 (Notes and Comments) X, 112

From Chancellor Day to Today: McGill and the 130 Years of the Quebec Civil Code (Notes and Comments) X, 110

(The) Gardens of the Lord: A Description of the Moravian Church in Labrador and the Lande Collection entitled The Moravian Missions to the Eskimos of Labrador II, 27

Gentlemanly Satisfaction: The Wellington-Winchilsea Duel of 1829 IX, 59

(The) General Portrayed: Sir Arthur Currie and His Painters VII, 73

Greenland Adventure: 1935. Diary of a Very Young Man, age 20 IV, 7

(The) Haemmerle Archive (Notes and Comments) V, 217

(Un) héritage métis (Notes and Comments) III, 151

Hidden Treasures at McGill: A Survey of Manuscripts and Historical Documents II, 65

(A) History and Description of the Burney Project I, (38)

History of the Polish Institute Library in Montreal (Notes and Comments) VI, 156

"House Portraits". Four Krieghoff Oil Paintings at the McCord Museum (Notes and Comments) VII, 164

Hugh MacLennan As I Knew Him VII, 113

Human Rights Inside the United Nations: The Humphrey Diaries, 1948-1959 IV, 143

Humphrey and the Old Revolution: Human Rights in the Age of Mistrust VIII, 121

Important Additions to the McCord's Furniture Collection (Notes and Comments) VI, 162

In Memoriam: John Peters Humphrey (1905-1995) VIII, 11

In Memoriam: Max Dunbar (1914-1995) VIII, 9

In Their Own Write: An Exhibition Celebrating 175 Years of McGill Authorship (Notes and Comments) IX, 118

In Touch With History. The Archival Collection of Professor Thomas Henry Clark (Notes and Comments) VII, 174

Isabella Christine McLennan VIII, 27

James McGill. An Album IX, 17

James McGill and the War of 1812 VII, 41

John Stephenson's Secret VIII, 137

John Wildman and Rushworth's Historical Collections: An Editor Identified? III, 109

Judge Robert MacKay's 1882 Catalogue of Books: A Preliminary Analysis X, 65

Leacock Enters McGill (Notes and Comments) VII, 149

(The) Leacock Mission Statement VII, 33 
Leacock Remains at McGill. Some Notes on the Stephen Leacock Collection VII, 11

(The) Legacy of Peter Redpath IX, 23

Macdonald Physics Building: 1893-1993 (Notes and Comments) VII, 155

Margaret Ridley Charlton, Medical Librarian and Historian: An Evaluation of Her Career II, 55

(The) McCord Museum's Inaugural Exhibition Programme (Notes and Comments) V, 223

McGill Law Library Moves into Cyberspace(s) (Notes and Comments) X, 115

(The) McGill University Collection of Greek and Roman Coins: New Evidence for its History IV, 109

(The) McGill University Library Catalogue of 1876: A Preliminary Statistical Analysis (Notes and Comments) IX, 120

McGill University on the Landscape of Canadian Higher Education: Historical Reflections X, 17

McGill's Nobel Laureate: The Rutherford Centenary X, 11

McGill's Second Kierkegaard Collection V, 173

McLennan Library: Twenty-Fifth Anniversary (Notes and Comments) VII, 152

Moses Maimonides: His Works and the McGill Collection I, 84

Moshe Safdie's Archive (Notes and Comments) IV, 183

(Le) mouvement de modernization des musées scientifiques au XX siècle: le cas du Musée Redpath de I'Université McGill III, 77

"Much Given to Reading". A Literary Footnote on James McGill IX, 9

Munificent, Wise and Thoughtful Gifts: Grace and Peter Redpath and the Redpath Tracts VI, 45

My Dear Eve ... The Letters of Ernest Rutherford to Arthur Eve, 1907-1908 I, 3

My Dear Eve ... The Letters of Emest Rutherford to Arthur Eve, 1909-1911 II, 111

My Dear Eve ... The Letters of Ernest Rutherford to Arthur Eve, 1912-1914 IV, 69

My Dear Eve ... The Letters of Ernest Rutherford to Arthur Eve, 1915-1919 V, 123

(The) Need for Dormitories at McGill VII, 7

Noel Buxton: The "Trouble Maker" and His Papers III, 131

Not a Bad "Impression": A Fine "Rembrandt" Print in McGill's Collection X, 35

Ottoman-Turkish Manuscripts in the Islamic Studies Library and Other Libraries of McGill University $\mathrm{X}, 41$

Percy Erskine Nobbs: Teacher and Builder of Arhitecture IX, 47

(The) Peter Redpath Museum, an Architectural Analysis I, (50)

(A) Portrait of Mary Todd Ferrier (Notes and Comments) IV, 179

Print Culture and English-Speaking Quebec X, 93

Pseudochristus: A Religious Romance, 1649-1650 VI, 97

Recent Additions to the Hume Collection (Notes and Comments) IV, 181

(The) Redpath Family and the McGill Libraries' Collections (Notes and Comments) VI, 143 
(The) Redpath Hall: The Portraits Vl, 85

Remembering War in Imperial Canada: David Ross McCord and The McCord National Museum IX, 97

(A) Remnant of a Gislenian Anthology I, 118

René Cassin and the Daughter of Time: The First Draft of the Universal Declaration of Human Rights II, 7

Rescue of Jews from Annihilation: Resistance and Responsibility in Nazi-Occupied Denmark VIII, 97

(A) Russian Diplomat in China: The Papers of Petr Genrikhovich Tiedemann at McGill (Notes and Comments) VIII, 147

(The) Rutherford Museum (Notes and Comments) VII, 183

(A) Samaritan Manuscript in McGill University V, 161

Scholar Librarians: Gould, Lomer and Pennington I, 95

(The) Sidney Dawes Collection at McGill University: A Catalogue V, 85

Silverware Crafted by Carl Poul Petersen: Recent Donation in the Decorative Arts Collection, McCord Museum of Canadian History (Notes and Comments) IX, 115

Sir William Macdonald: An Unfinished Portrait VIII, 59

Some Thoughts on Fontanus, from the Collections of McGill University X, 9

Son of the Great Dominion: W.D. Lighthall and the Lighthall Family Papers II, 103

Sources for Tudor and Stuart History: The William Clarke Papers I, 59

Sources of Civil Law: The Wainright Collection I, 77

Stephen Leacock and His Books VII, 15

"That Best Portion of a Good Woman's Life": Gertrude Mudge, 1886-1958 VIII, 49

Under the Banner of the Book: The Work of Karel Teige IV, 125

Views of Rome, Visions of Rome. Piranesi Prints from the Nobbs Collection in the BlackaderLauterman Library, McGill University (Notes and Comments) VII, 158

Venice and Crete: Documents from the XVIth to XVIIIth Centuries IV, 55

(The) Voyage of Giovanni da Verrazzano: A Newly Discovered Manuscript V, 7

Walter Hitschfeld Geographic Information Centre (Notes and Comments) X, 116

"When There Is No Vision the People Perish." The McCord Family Papers, 1766-1945 III, 17

Women in the University: The Fourth Phase VIII, 13

Witness Testimonies of the Holocaust and the Meaning of Memory VII, 103 


\section{FONTANUS SUBJECT INDEX}

Abbott, Maude See Maude Abbott

al-Ghâfiqî. Arabic Manuscripts. Arabic Calligraphy. Herbal. II, 37

Alfred Haemmerle. Haemmerle Archive. Letters. McGill University Archives. V, 217

Alice E. Johannsen. E. Lionel Judah. Science Museums. Redpath Museum. III, 77

Amy Haemmerle. Haemmerle Archive. Letters. McGill University Archives. V, 217

Anatole Haemmerle. Haemmerle Archive. Letters. McGill University Archives. V, 217

Andrew Taylor. Redpath Library. Peter Redpath. VI, 69

Anglophone Contributions. Printing and Publishing. Québec Province. X, 93

Arabic Calligraphy. Herbal. al-Ghâfiqî. Arabic Manuscripts. II, 37

Arabic Manuscripts. Arabic Calligraphy. Herbal. al-Ghâfiqî. II, 37

Arabic Manuscripts. Blacker-Wood. Library. II, 37

Arabic Manuscripts. Islamic Studies Library. II, 37

Arabic Manuscripts. McLennan Library. II, 37

Arabic Manuscripts. Osler Library. II, 37

Arabic Manuscripts. Rare Books and Special Collections Division. II, 37

Arabic Manuscripts. Rare Books and Special Collections Division. Qur'ân. Codices. IV, 35

Arabic Manuscripts. Rare Books and Special Collections Division. Qur'ân. Fragments. III, 35

Architectural Legacy. Percy Erskine Nobbs. IX, 47

Art Cabinet. McCord Museum. Herter Brothers. VI, 162

Arthur Eve See Ernest Rutherford. Letters.

Arthur Wellesley (1st Duke of Wellington). George William Finch-Hatton (9th Earl of Winchilsea).

Duel. IX, 59

Austin "Dink" Carroll. Papers. McGill University Archives. VI, 149

Authors. McGill University Affiliation. Exhibition. IX, 118

Beatrice V. Simon. Memorial. VII, 181

Blackader-Lauterman Library. Percy Nobbs Collection. Piranesi. Prints. VII, 158

Blacker-Wood Library. Arabic Manuscripts. II, 37

Book Design. Modernism. Devětsil Group. Karel Teige. IV, 125

Book Jackets. Rare Books and Special Collections Division. Stephen Leacock. Works. VII, 15

Book of the Hours. Medieval Manuscript. Officium Beate Virginis Mariae. VIII, 109

British Parliamentary Papers. Second Language Teaching. I, 105

Burney, Fanny See Fanny Burney 
Burney Project. Fanny Burney. I, (38b)

Buxton-Noel, Noel See Noel Noel-Buxton

Calligraphy. Arabic. Arabic Manuscripts. Herbal. al-Ghâfiqî. II, 37

Canadian Architecture Collection. Rare Books and Special Collections Division. Moshe Safdie Archive. IV, 183

Canadian Art Collection. McGill Museum of Canadian Art. Sidney Dawes. V, 73

Canadian Unity and Quebec. Radio Script. Hugh MacLennan. John Humphrey. Émile Vaillancourt. VI, 119

Carl Poul Petersen. Silverware Collection. McCord Museum. IX, 115

Carroll, Austin "Dink" See Austin "Dink" Carroll

Cassin, René See René Cassin

Castelli, Pietro See Pietro Castelli

Cattaneo Family. Venice. Crete. Manuscripts. Rare Books and Special Collections Division. IV, 55

Ceramic Collection. Weathervanes. McCord Museum. Charles deVopli. III, 153

Charles deVopli. Ceramic Collection. Weathervanes. McCord Museum. III, 153

Charles Gould. Gerhard Lomer. Richard Pennington. I, 95

Charlton, Margaret Ridley See Margaret Ridley Charlton

Children's Books. McGill University Libraries. IV, 175

China. Petr Genrikhovich Tidemann. Russian Diplomat. Papers. McGill University Archives. VIII, 147

Civil Law. Wainwright Collection. Nahum Gelber Law Library. I, 77

Clark, Thomas Henry See Thomas Henry Clark

Coins See Numismatic Collection

Concert. Montreal, 1786. Earliest Music Programme. Rare Books and Special Collections Division. V, 235

Content Analysis. Personal Library. Robert MacKay (Judge). X, 65

Cornelius Krieghoff. "House Portraits". McCord Museum. VII, 164

Crete. Venice . Manuscripts. Cattaneo Family. Rare Books and Special Collections Division. IV, 55

Crete. Venice. Manuscripts. Michieli Family. Rare Books and Special Collections Division. IV, 55

Crete. Venice. Manuscripts. Mormori Family. Rare Books and Special Collections Division. IV, 55

Cultural Diversity. Human Rights. F.R. Scott. On Kanbawza Road. X, 81

Currie, Sir Arthur See Sir Arthur Currie

Danish Collection. McGill Libraries. IX, 131

David Hume Collection. Rare Books and Special Collections Division. IV, 181

David Ross McCord. Family Papers. McCord Museum. III, 17

David Ross McCord. Military History Collection. McCord Museum. IX, 97 
Dawes, Sidney See Sidney Dawes.

Dawson, Sir William See Sir William Dawson

Denmark. Jews. Rescue. VIII, 97

Department of Rare Books and Special Collections See Rare Books and Special Collections Division.

(The) Descent from the Cross (Print). Rembrandt. Authentication. Rare Books and Special Collections Division. X, 35

Devětsil Group. Karel Teige. Book Design. Modernism. IV, 125

deVolpi, Charles See Charles deVolpi.

Diary. Greenland Expedition. Max J. Dunbar. IV, 7

Duel. Arthur Wellesley (1st Duke of Wellington). George William Finch-Hatton (9th Earl of Winchilsea). IX, 59

Dunbar, Max J. See Max J. Dunbar.

Duncan McNab McEachran. Montreal Veterinary College. McGill University. X, 101

E. Lionel Judah. Alice E. Johannsen. Science Museums. Redpath Museum. III, 77

Earliest Music Programme. Rare Books and Special Collections Division. Broadside. Montreal, 1786. V, 235

Early Canadian Herbal, 1625. Plant Book. Pietro Castelli. Rare Books and Special Collections Division. VII, 170

Early Children's Books. McGill University Libraries. IV, 175

Egypt Exploration Fund. John Garstang. Egyptian Artifacts. Nubian Artifacts. Redpath Museum. III, 117

Egyptian Artifacts. Nubian Artifacts. Redpath Museum. Egypt Exploration Fund. John Garstang. III, 117

Ellis, Humphrey See Humphrey Ellis.

Émile Vaillancourt. Canadian Unity and Quebec. Radio Script. Hugh MacLennan. John Humphrey. VI, 119

English Civil War. Historical Collections. James Rushworth. John Wildman. III, 109

Ernest Rutherford. X, 11

Ernest Rutherford. Rutherford Museum. Letters. I, (3); II, 111; IV, 69, V, 123

Eve, Arthur See Ernest Rutherford. Letters.

Exploration. Manuscript. Giovanni da Verrazzano. Rare Books and Special Collections Division. V, 7

F. R. Scott. Letter from John Humphrey. McGill University Archives. X, 112

F.R. Scott. Literary Influence. III, 17

F.R. Scott. On Kanbawza Road. Cultural Diversity. Human Rights. X, 81

F.R. Scott. Personal Library. Rare Books and Special Collections Division. II, 97

Faculty of Music. Redpath Hall. VI, 147

Fanny Burney. Burney Project. I, (38b). 
Ferrier, Mary Todd See Mary Todd Ferrier

Finch-Hatton, George William See George William Finch-Hatton (9th Earl of Winchilsea)

Fontanus. Inception. $X, 9$

Frank Cyril James. James Papers. McGill University Archives. III, 65

Franklin, William See William Franklin

Garstang, John See John Garstang

George William Finch-Hatton (9th Earl of Winchilsea). Arthur Wellesley (1st Duke of Wellington). Duel. IX, 59

Gerhard Lomer. Richard Pennington. Charles Gould. I, 95

Gertrude Mudge VIII, 49

Giovanni da Verrazzano. Rare Books and Special Collections Division. Exploration. Manuscript. V, 7

Gislenus. Manuscript. I, (117b)

Gould, Charles See Charles Gould

Grace Redpath. Peter Redpath. Redpath Hall. Portraits. VI, 85

Grace Redpath. Peter Redpath. Redpath Tracts. Rare Books and Special Collections Division. VI, 45

Greek and Roman Coins. Numismatic Collection. Provenance. Natural History Society of Montreal. IV, 109

Greek and Roman Coins. Numismatic Collection. Provenance. Redpath Museum. IV, 109

Greenland Expedition. Max J. Dunbar. Diary. IV, 7

Haemmerle, Alfred See Haemmerle Archive

Haemmerle, Amy See Haemmerle Archive

Haemmerle, Anatole See Haemmerle Archive

Haemmerle Archive. Letters. McGill University Archives. Alfred Haemmerle. V, 217

Haemmerle Archive. Letters. McGill University Archives. Amy Haemmerle. V, 217

Haemmerle Archive. Letters. McGill University Archives. Anatole Haemmerle. V, 217

Health Sciences Library. Margaret Ridley Charlton. II, 55

Herbal. al-Ghâfiqî. Arabic Manuscripts. Arabic Calligraphy. II, 37

Herter Brothers. Art Cabinet. McCord Museum. VI, 162

Historical Collections. James Rushworth. John Wildman. English Civil War. III, 109

Holocaust. "Living Testimonies". Oral History. Video Archive. Rare Books and Special Collections Division. V, 229

Holocaust. Witness Testimonies. Oral History. Video Archive. Rare Books and Special Collections Division. VII, 103

House Portraits. Cornelius Krieghoff. McCord Museum. VII, 164

Hugh MacLennan. Hugh MacLennan Memorial Lecture (1993). VII, 113 
Hugh MacLennan. John Humphrey. Émile Vaillancourt. Canadian Unity and Quebec. Radio Script. VI, 119

Hugh MacLennan Memorial Lecture (1993). Hugh MacLennan. VII, 113

Human Rights. Cultural Diversity. F.R. Scott. On Kanbawza Road. X, 81

Hume, David See David Hume

Humphrey Ellis. Redpath Tract. William Franklin. Trial. Pseudochristus. VI, 97

Humphrey, John Peters See John Humphrey

Isabella Christine McLennan. VIII, 27

Islamic Studies Library. Arabic Manuscripts. II, 37

Islamic Studies Library. Ottoman-Turkish Manuscripts. Rare Books and Special Collections Division. $\mathrm{X}, 41$

James, Frank Cyril See Frank Cyril James

James McGill. Reading Habits. IX, 9

James McGill. War of 1812. VII, 41

James Papers. McGill University Archives. Frank Cyril James. III, 65

James Rushworth. John Wildman. English Civil War. Historical Collections. III, 109

Jews. Rescue. Denmark. VIII, 97

Johannsen, Alice E. See Alice E. Johanssen

John Garstang. Egyptian Artifacts. Nubian Artifacts. Redpath Museum. Egypt Exploration Fund. III, 117

John Humphrey. Diaries. McGill University Archives. Political Associates. VIII, 121

John Humphrey. Diaries. Universal Declaration of Human Rights. United Nations. McGill University Archives. IV, 143

John Humphrey. Émile Vaillancourt. Canadian Unity and Quebec. Radio Script. Hugh MacLennan. VI, 119

John Humphrey. Letter to F. R. Scott. McGill University Archives. X, 112

John Humphrey. Memorial. VIII, 11

John Humphrey. Political Associates. Diaries. McGill University Archives. VIII, 121

John Humphrey. Universal Declaration of Human Rights. United Nations. McGill University Archives. IV, 143

John Stephenson. VIII, 137

John Wildman. English Civil War. Historical Collections. James Rushworth. III, 109

Judah, E. Lionel See E. Lionel Judah

Karel Teige. Book Design. Modernism. Devetsil Group. IV, 125

Kierkegaard, Søren See Søren Kierkegaard

Krieghoff, Cornelius See Cornelius Krieghoff 
Labrador. Moravian Missions. Lande Eskimo Collection. II, 27

Lande Eskimo Collection. Labrador. Moravian Missions. II, 27

Leacock, Stephen See Stephen Leacock

Library Catalogue, 1876. Redpath Library. Statistical Analysis. IX, 120

Lighthall, William Douw See William Douw Lighthall

Living Testimonies See also Witness Testimonies

"Living Testimonies". Oral History. Video Archive. Rare Books and Special Collections Division.

Holocaust. V, 229

Lomer, Gerhard See Gerhard Lomer

Macdonald Physics Building. Centennial. VII, 155

Macdonald, Sir William See Sir William Macdonald

MacLennan, Hugh See Hugh MacLennan

MacKay, Robert See Robert MacKay (Judge)

Maimonides, Moses See Moses Maimonides

Manuscripts. McGill Libraries. Survey. II, 65

Margaret Ridley Charlton. Health Sciences Library. II, 55

Marvin Duchow Music Library. Thomas Wesley Mills. Book Bequest. Violin Preservation. III, 35

Mary Todd Ferrier. Portrait. McCord Museum. IV, 179

Maude Abbott. McGill Museum of Pathology. McGill University. X, 71

Max J. Dunbar. Diary. Greenland Expedition. IV, 7

Max J. Dunbar. Memorial. VIII, 9

McCord, David Ross. See David Ross McCord

McCord Museum of Canadian History See McCord Museum

McCord Museum. 350th Anniversary. Inaugural Exhibition (May1992) V, 223

McCord Museum. Art Cabinet. Herter Brothers. VI, 162

McCord Museum. Carl Poul Petersen. Silverware Collection. IX, 115

McCord Museum. Charles deVopli. Ceramic Collection. Weathervanes. III, 153

McCord Museum. Cornelius Krieghoff. "House Portraits". VII, 164

McCord Museum. David Ross McCord. Family Papers. III, 17

McCord Museum. David Ross McCord. Military History Collection. IX, 97

McCord Museum. Herter Brothers. Art Cabinet. VI, 162

McCord Museum. Mary Todd Ferrier. Portrait. IV, 179

McCord Museum. Molson Family Documents. I, 69

McCord Museum. Notman Photographic Archives. William Notman. VII, 127

McCord Museum. William Notman. Notman Photographic Archives. VII, 127 
McEachran, Duncan McNab See Duncan McNab McEachran

McGill, James See James McGill

McGill Law Faculty. Québec Civil Code. X, 110

McGill Museum of Canadian Art. Sidney Dawes. Canadian Art Collection. V, 73

McGill Museum of Pathology. McGill University. Maude Abbott. X, 71

McGill University. Higher Education. Historical Role. X, 17

McGill University. McGill Museum of Pathology. Maude Abbott. X, 71

McGill University. Montreal Veterinary College. Duncan McNab McEachran. X, 101

McGill University. Residences. VII, 7

McGill University. Science and Scientists. IX, 105

McGill University. Women's Role and Contributions. VIII, 13

McGill University Archives. Austin "Dink" Carroll. Papers. VI, 149

McGill University Archives. Diaries, 18th-20th Century. V, 33

McGill University Archives. Frank Cyril James. James Papers. III, 65

McGill University Archives. Haemmerle Archive. Letters. V, 217

McGill University Archives. John Humphrey. Diaries. Political Associates. VIII, 121

McGill University Archives. John Humphrey. Diaries. Universal Declaration of Human Rights. United Nations. IV, 143

McGill University Archives. John Humphrey. Letter to F. R. Scott. X, 112

McGill University Archives. Petr Genrikhovich Tidemann. Russian Diplomat. China. Papers. VIII, 147

McGill University Archives. Royal Institute for the Advancement of Learning. Correspondence. V, 55

McGill University Archives. Sir William Dawson. Papers. IX, 125

McGill University Archives. Stephen Leacock. William Peterson. Letters. VII, 149

McGill University Archives. Thomas Henry Clark Collection. Paleontology. VII, 174

McGill University Archives. William Peterson. Stephen Leacock. Letters. VII, 149

McGill University in Satirical Fiction. IX, 29

McGill University Libraries. Collections. Redpath Family. VI, 143

McGill University Libraries. Danish Collection. IX, 131

McGill University Libraries. Early Children's Books. IV, 175

McGill University Libraries. Manuscripts. Survey. II, 65

McLennan, Isabella Christine See Isabella Christine McLennan

McLennan Library. Arabic Manuscripts. II, 37

McLennan Library. Moses Maimonides. I, 84

McLennan Library. Twenty-Fifth Anniversary. VII, 152

Medieval Manuscript. Book of the Hours. Officium Beate Virginis Mariae. VIII, 109 
Meroitic Cylinders. Palaeolithic Sculptures. Redpath Museum. Archaeological Collections. VII, 53

Michieli Family. Venice. Crete. Manuscripts. Rare Books and Special Collections Division. IV, 55

Military History Collection. David Ross McCord. McCord Museum. IX, 97

Mills, Thomas Wesley See Thomas Wesley Mills

Modernism. Devětsil Group. Karel Teige. Book Design. IV, 125

Molson Family Documents. McCord Museum. I, 69

Montreal Veterinary College. McGill University. Duncan McNab McEachran. X, 101

Moravian Missions. Labrador. Lande Eskimo Collection. II, 27

Mormori Family. Venice. Crete. Manuscripts. Rare Books and Special Collections Division. IV, 55

Moses Maimonides. McLennan Library. Osler Library. Rare Books and Special Collections Division.

I, 84

Moshe Safdie Archive. Canadian Architecture Collection. Rare Books and Special Collections Division. IV, 183

Mudge, Gertrude See Gertrude Mudge

Nahum Gelber Law Library. X, 115

Nahum Gelber Law Library. Universal Declaration of Human Rights. Manuscript. René Cassin. II, 7

Nahum Gelber Law Library. Wainwright Collection. Civil Law. I, 77

Natural History Society of Montreal. Numismatic Collection. Provenance. Greek and Roman Coins. IV, 109

Nobbs, Percy See Percy Nobbs

Noel Noel-Buxton. Papers. Rare Books and Special Collections Division. III, 131

Notman Photographic Archives. William Notman. McCord Museum. VII, 127

Notman, William See William Notman

Nubian Artifacts. Redpath Museum. Egypt Exploration Fund. John Garstang. Egyptian Artifacts. III, 117

Numismatic Collection. Provenance. Greek and Roman Coins. Natural History Society of Montreal. IV, 109

Numismatic Collection. Provenance. Greek and Roman Coins. Redpath Museum. IV, 109

Officium Beate Virginis Mariae. Book of the Hours. Medieval Manuscript. VIII, 109

On Kanbawza Road. Human Rights. Cultural Diversity. F.R. Scott. X, 81

Oral History. Video Archive. Rare Books and Special Collections Division. Holocaust. "Living

Testimonies". V, 229

Oral History. Video Archive. Rare Books and Special Collections Division. Holocaust. Witness

Testimonies. VII, 103

Osler Library. Arabic Manuscripts. II, 37

Osler Library. Moses Maimonides. I, 84

Osler Library. Ottoman-Turkish Manuscripts. Islamic Studies Library. X, 41 
Ottoman-Turkish Manuscripts. Islamic Studies Library. Osler Library. X, 41

Ottoman-Turkish Manuscripts. Islamic Studies Library. Rare Books and Special Collections Division. $\mathrm{X}, 41$

Ottoman-Turkish Manuscripts. Osler Library. Rare Books and Special Collections Division. X, 41 Palaeolithic Sculptures. Redpath Museum. Archaeological Collections. Meroitic Cylinders. VII, 53

Paleontology. Thomas Henry Clark Collection. McGill University Archives. VII, 174

Pennington, Richard See Richard Pennington

Pentateuch. Manuscript. Rare Books and Special Collections Division. Samaritans. V, 161

Percy Erskine Nobbs. Architectural Legacy. IX, 47

Percy Erskine Nobbs Collection. Piranesi. Prints. Blackader-Lauterman Library. VII, 158

Personal Library. F.R. Scott. II, 97

Personal Library. Robert MacKay (Judge). Content Analysis. X, 65

Peter Redpath. Andrew Taylor. Redpath Library. VI, 69

Peter Redpath. Grace Redpath. Redpath Tracts. Rare Books and Special Collections Division. VI, 45

Peter Redpath. Memorial. VI, 7

Peter Redpath. Redpath Hall. Portraits. VI, 85

Peter Redpath. Redpath Library. VI, 7; IX, 23

Peter Redpath Museum See Redpath Museum

Petersen, Carl Poul See Carl Poul Petersen

Petr Genrikhovich Tidemann. Russian Diplomat. China. Papers. McGill University Archives. VIII, 147

Pietro Castelli. Early Canadian Herbal, 1625. Plant Book. Rare Books and Special Collections Division. VII, 170

Piranesi. Prints. Blackader-Lauterman Library. Percy Nobbs Collection. VII, 158

Plant Book. Pietro Castelli. Early Canadian Herbal, 1625. Rare Books and Special Collections Division. VII, 170

Polish Institute. Library. Montreal. History. VI, 156

Printing and Publishing. Québec Province. Anglophone Contributions. X, 93

Pseudochristus. Humphrey Ellis. Redpath Tract. William Franklin. Trial. VI, 97

Publishing and Printing. Québec Province. Anglophone Contributions. X, 93

Québec Civil Code. McGill Law Faculty Contribution. X, 110

Québec Province. Printing and Publishing. Anglophone Contributions. X, 93

Qur'ân. Arabic Manuscripts. Codices. Rare Books and Special Collections Division. IV, 35

Qur'ân. Arabic Manuscripts. Fragments. Rare Books and Special Collections Division. III, 45

Radio Script. Hugh MacLennan. John Humphrey, Émile Vaillancourt. Canadian Unity and Quebec. VI, 119

Rare Books and Special Collections Division. Arabic Manuscripts. II, 37, III, 35, IV, 35 
Rare Books and Special Collections Division. Book of the Hours. Medieval Manuscript. VIII, 109 Rare Books and Special Collections Division. Canadian Architecture Collection. Moshe Safdie Archive. IV, 183

Rare Books and Special Collections Division. Cattaneo Family. Venice. Crete. Manuscripts. IV, 55

Rare Books and Special Collections Division. David Hume Collection. IV, 181

Rare Books and Special Colections Division. (The) Descent from the Cross (Print). Rembrandt. Authentication. X, 35

Rare Books and Special Collections Division. Earliest Music Programme. Broadside. Montreal, 1786. V, 235

Rare Books and Special Collections Division. F.R. Scott. Personal Library. II, 97

Rare Books and Special Collections Division. Giovanni da Verrazzano. Exploration. Manuscript. V, 7

Rare Books and Special Collections Division. Gislenus Manuscript. I, (117b)

Rare Books and Special Collections Division. Henry Hardinge (1st Viscount Hardinge) Papers.

Wellington. Duel. IX, 59

Rare Books and Special Collections Division. Lande Eskimo Collection. II, 27

Rare Books and Special Collections Division. "Living Testimonies". Video Archive. Holocaust. Oral History. V, 229

Rare Books and Special Collections Division. Michieli Family. Venice. Crete. Manuscripts. IV, 55

Rare Books and Special Collections Division. Molson Family Documents. I, 69.

Rare Books and Special Collections Division. Mormori Family. Venice. Crete. Manuscripts. IV, 55

Rare Books and Special Collections Division. Moses Maimonides. I, 84

Rare Books and Special Collections Division. Moshe Safdie Archive. Canadian Architecture Collection. IV, 183

Rare Books and Special Collections Division. Noel Noel-Buxton. Papers. III, 131

Rare Books and Special Collections Division. Officium Beate Virginis Mariae. Book of the Hours. VIII, 109

Rare Books and Special Collections Division. Ottoman-Turkish Manuscripts. X, 41

Rare Books and Special Collections Division. Plant Book. Pietro Castelli. Early Canadian Herbal,1625. VII, 170

Rare Books and Special Collections Division. Qur'ân. Codices. IV, 35

Rare Books and Special Collections Division. Qur'ân. Fragments. III, 35

Rare Books and Special Collections Division. Redpath Tract. Pseudochristus. Humphrey Ellis. VI, 97

Rare Books and Special Collections Division. Redpath Tracts. Grace Redpath. Peter Redpath. VI, 45

Rare Books and Special Collections Division. Rembrandt. The Descent from the Cross (Print).

Authentication. X, 35

Rare Books and Special Collections Division. Samaritans. Pentateuch. Manuscript. V, 161

Rare Books and Special Collections Division. William Douw Lighthall Papers. II, 103 
Rare Books and Special Collections Division. Witness Testimonies. Video Archive. Holocaust. Oral History. VII, 103

Redpath Family. McGill Libraries. Collections. VI, 143

Redpath, Grace See Grace Redpath

Redpath Hall. Faculty of Music. VI, 147

Redpath Hall. Portraits. VI, 85

Redpath Library. VI, 7; IX, 23

Redpath Library. Library Catalogue, 1876. Statistical Analysis. IX, 120

Redpath Library. Peter Redpath. Andrew Taylor. VI, 69

Redpath Museum. Alice E. Johannsen. E. Lionel Judah. III, 77

Redpath Museum. Archaeoloical Collections. Meroitic Cylinders. Palaeolithic Sculptures. VII, 53

Redpath Museum. Architectural Analysis. I, (49b)

Redpath Museum. E. Lionel Judah. Alice E. Johannsen. III, 77

Redpath Museum. Egypt Exploration Fund. John Garstang. Egyptian Artifacts. Nubian Artifacts. III, 117

Redpath Museum. Meroitic Cylinders. Palaeolithic Sculptures. Archaeological Collections. VII, 53

Redpath Museum. Molson Family Documents. I, 69

Redpath Museum. Numismatic Collection. Provenance. Greek and Roman Coins. IV, 109

Redpath Museum. Palaeolithic Sculptures. Archaeological Collections. Meroitic Cylinders. VII, 53

Redpath Museum. Science Museums. E. Lionel Judah. Alice E. Johanssen. III, 77

Redpath Museum. William Clarke. Papers. I, 59

Redpath, Peter. See Peter Redpath

Redpath Tract. William Franklin. Trial. Pseudochristus. Humphrey Ellis. VI, 97

Redpath Tracts. Rare Books and Special Collections Division. Grace Redpath. Peter Redpath. VI, 45

Rembrandt. The Descent from the Cross (Print). Authentication. Rare Books and Special Collections Division. X, 35

René Cassin. Universal Declaration of Human Rights. Manuscript. Nahum Gelber Law Library. II, 7

Robert MacKay (Judge). Personal Library. Content Analysis. X, 65

Richard Pennington. Charles Gould. Gerhard Lomer. I, 95

Royal Institute for the Advancement of Learning. Correspondence. McGill University Archives. V, 55

Rushworth,James. See James Rushworth

Russian Diplomat. Petr Genrikhovich Tidemann. China. Papers. McGill University Archives. VIII, 147

Rutherford, Ernest See Ernest Rutherford

Rutherford Museum. VII, 183

Rutherford Museum. Ernest Rutherford. Letters. I, (3); II, 111; IV, 69; V, 123 
Safdie, Moshe See Moshe Safdie

Samaritans. Pentateuch. Manuscript. Rare Books and Special Collections Division. V, 161

Satirical Fiction McGill University in IX, 29

Science and Scientists. McGill University. IX, 105

Science Museums. Redpath Museum. E. Lionel Judah. Alice E. Johannsen. III, 77

Scientists and Science. McGill University. IX, 105

Scott, F.R. See F.R. Scott

Second Language Teaching. British Parliamentary Papers. I, 105

Sidney Dawes. Canadian Art Collection. McGill Museum of Canadian Art. V, 73

Silverware Collection. Carl Poul Petersen. McCord Museum. IX, 115

Simon, Beatrice V. See Beatrice V. Simon

Sir Arthur Currie. Portraits. VII, 73

Sir William Dawson. Papers. McGill University Archives. IX, 125

Sir William Macdonald. VIII, 59

Søren Kierkegaard. Samlede Voerker (Collected Works). Text Analysis. Sofware. V, 173

Statistical Analysis. Library Catalogue, 1876. Redpath Library. IX, 120

Stephen Leacock. Letters. William Peterson. McGill University Archives. VII, 149

Stephen Leacock. Mission Statement. VII, 33

Stephen Leacock. Works. Book Jackets. Rare Books and Special Collections Division. VII, 15

Stephenson, John See John Stephenson

Taylor, Andrew See Andrew Taylor

Teige, Karel See Karel Teige

Text Analysis. Sofware. Søren Kierkegaard. Samlede Voerker (Collected Works). V, 173

Thomas Henry Clark Collection. Paleontology. McGill University Archives. VII, 174

Thomas Wesley Mills. Book Bequest. Violin Preservation. Marvin Duchow Music Library. III, 35

Tidemann, Petr Genrikhovich See Petr Genrikhovich Tidemann

Trial. Pseudochristus. Humphrey Ellis. Redpath Tract. William Franklin. VI, 97

Tudor and Stuart History. William Clarke. Papers. I, 59

Turkish Manuscripts See Ottoman-Turkish Manuscripts

United Nations. Diaries. McGill University Archives. John Humphrey. Universal Declaration of Human Rights. IV, 143

United Nations. Manuscript. Nahum Gelber Law Library. René Cassin. Universal Declaration of Human Rights. II, 7

Universal Declaration of Human Rights. United Nations. Diaries. McGill University Archives. John Humphrey. IV, 143 
Universal Declaration of Human Rights. United Nations. Manuscript. Nahum Gelber Law Library. René Cassin. II, 7

Vaillancourt, Émile See Émile Vaillancourt

Venice. Crete. Manuscripts. Cattaneo Family. Rare Books and Special Collections Division. IV, 55

Venice. Crete. Manuscripts. Michieli Family. Rare Books and Special Collections Division. IV, 55

Venice. Crete. Manuscripts. Mormori Family. Rare Books and Special Collections Division. IV, 55

Verrazzano, Giovanni da See Giovanni da Verrazzano

Video Archive. Oral History. Rare Books and Special Collections Division. Holocaust. "Living Testimonies". V, 229

Video Archive. Oral History. Rare Books and Special Collections Division. Holocaust. Witness Testimonies. VII, 103

Violin Preservation. Marvin Duchow Music Library. Thomas Wesley Mills. Book Bequest. III, 35

Wainwright Collection. Civil Law. Nahum Gelber Law Library. I, 77

Walter Hitschfeld Geographic Information Centre. X, 116

War of 1812. James McGill. VII, 41

Weathervanes. McCord Museum. Charles deVopli. Ceramic Collection. III, 153

Wellesley, Arthur See Arthur Wellesley (1st Duke of Wellington)

Wildman, John See John Wildman

William Douw Lighthall. Papers. Rare Books and Special Collections Division. II, 103

William Franklin. Trial. Pseudochristus. Humphrey Ellis. Redpath Tract. VI, 97

William Notman. Notman Photographic Archives. McCord Museum. VII, 127

William Peterson. Stephen Leacock. Letters. McGill University Archives. VII, 149

Witness Testimonies. Oral History. Video Archive. Rare Books and Special Collections Division. Holocaust. VII, 103

Women's Role and Contributions. McGill University. VIII, 13

\section{FONTANUS ILLUSTRATION INDEX}

\section{INDIVIDUALS AND GROUP}

Abbott, Maud (Photo) VIII, 18

Atter, Michael (Photos) IV, 10,13, 20

Ayotte, A (Photo) III, 83

Beatty, Sir William Edward (Portrait) VI, 93; (Photo) X, 20

Cappelørn Niels Jørgen (Photo) V, 210

Carroll, Austin "Dink" (Photos) VI, 150, 154 
Cassin, René (Photo) IV, 157

Chambers, Gretta (Photo) VIII, 22

Charlton, Margaret Ridley (Photo) II, 57

Clark, Thomas Henry (Photos) VII, 176, 179

Currie, Sir Arthur (Portraits) VI, 90; VII, 75, 76, 81, 85, 89, 91, (Charcoal Portrait) 80, (Pencil Drawing)

84; (Photos) VII, 93; IX, 39; X, 20 (on War publicity booklet)

Davis, Mortimer (Photo) VII, 137

Dawes, A. Sidney (Photo) V, 75

Dawson, John William (Portrait) VI, 90; (Photo) IX, 106, (Daguerrotype) 126, (Pencil sketch) 126

Derik, Carrie (Photo) VIII, 16

Douglas, Lewis William (Photo) X, 23

Dulaney, Isabella McMeekin (Photo) VIII, 43

Dunbar, Max (Photos) IV, 10; VIII, 8

Du Roure, Réné (Caricature) IX, 35

Eve, Arthur Stewart (Photos) IV, 73; V, 126; 151, 158

Eve, Cicely (Photo) V, 126

Eve, Elizabeth (Photo) V, 126

Eve, Joan (Photo) V, 126

Eve, Richard (Photo) V, 126

Ferrier, James (Portrait) VI, 93

Ferrier, Mary Todd (Portrait) IV, 179

Findlayson, Stuart (Portrait) VI, 93

Freedman, Samuel O. (Photo) IV, (4)

Garff, Joakim (Photo) V, 210

Geddes, Sir Aukland Campbell (Photo) X, 19

General Officers of World War I (Painting) VII, 86

Genrikhovich, Petr (Photo) VIII, 139

Gould, Charles Henry (Drawing) I, (96a); (Caricature) VI, 31

Gros, Louis (Photo) IV, 152

Gymnasts (Photo) VII, 139

Haemmerle, Alma (Photo) V, 221

Haemmerle, Amy Waht (Photo) V, 219

Haemmerle, Anatole (Photos) V, 219, 220

Haemmerle, Anatole Alfred (Photo) V, 219

Haemmerle, Irma (Photo) V, 221 
Hayward, Henry (Photos) IV, 10, 20

Heaphy, Phyllis (Photo) VIII, 22

Hogue, Stéphan (Photo) V, 210

Holtved, "The Professor" (Photo) IV, 20

Humphrey, John Peters (Photos) II, 9; IV, 147, 152, 157, 161; (Drawing) VI, 125; (Photo) VIII, 10

Hunt, T. Sterry (Photo) IX, 106

James, Frank Cyril (Photos) III, 66; V, 75; (Portrait) VI, 90; (Photo) X, 26

Johannsen, Alice E (Photos) III, 91, 97

Judah, Bernard Samuel (Portrait) V, 36

Judah, E. Lionel (Photos) III, 83, 87

Jukier, Rosalie (Photo) VIII, 23

Krivossidis, Nikos (Photo) V, 210

Lacoste, Francis (Photo) I, (79b)

Laugier, Henri (Photo) IV, 152

Leacock, Stephen (Photo) VII, (6), 10, (Caricature, pen/ink) 9, (Sketch/Photo) 34

Lees, Victoria (Photo) VIII, 23

Lighthall W.D. (Photo) II, 105

Lin, Mousheng (Photo) IV, 157

Lindsay, Mrs. W. B. (Photo) VII, 136

Lomer, Gerhard R. (Photo) I, (97b)

Maass, Otto (Photo) IX, 108

Macdonald Physics Laboratory (Staff Group Photo) I, 5; (Staff Group Photo) II, 113

Macdonald, William (Portrait) VI, 87; (Photo) VIII, 58; (Painting) 76

MacLennan, Hugh (Portrait) VI, 120; (Photos) 150; VII, 115

Malik, Charles (Photo) IV, 147

Manchester University, Physics Department (Group Photo) IV, 70

McConnell, John W. (Portrait) VI, 87

McCord, Anne Ross (Portrait) III, 27

McCord, David Ross (Portrait) III, 19

McCord, John Samuel (Portrait) III, 24

McEachran, Duncan, McNab (Photo) X, 105

McGill, James (Portrait) VI, 87; (Portrait) VII, 43; (Repro. Watercolour) IX, 19; (Portrait) IX, 20,

(Monument/Tomb) 22, (Bronze Sculpture) 22

McKinnon, Alastair (Photo) V, 210 
McLennan, Bartlett (Photo) VIII, 39

McLennan, Hugh (Photos) VIII, 28, 35.

McLennan, Isabella Christine (Photos) VII, 29, 31, 33, 36, 37, 43

McMeekin, Isabella Stewart McLennan (Photo) VIII, 43

Meredith, W.C.J. (Photo) I, (79b)

Mills, Thomas Wesley (Photo) III, 39, (Caricature) 41

Moller, Hans (Photo) X, (8)

Molson, Mrs. John (Photo) I, (71b)

Molson, William (Portrait) VI, 87

Morgan, Arthur Eustace (Photo) X, 23

Mott, Peter (Photos) IV, 10, 20

Mountain, G. J. (Photo) VI, 90

Mudge, Gertrude (Photo) VIII, 48

Murray, E. G. D. (Photo) IX, 112

Nobbs, Percy Erskine (Photos) IX, 46, 49, 92

Noel-Buxton, Lord Noel (Portrait) III, 133, (Photo) 145

Notman, Charles (Photos) VII, 128, 130

Notman, George (Photo) VII, 128

Notman, William (Photo) VII, 128

Notman, William McFadden (Photo) VII, 128

O'Donnell, Hugh (Photo) I, (79b)

Pennington, Richard (Photo) I, (99b)

Peterson, Sir William (Painting) VIII, 76; (Photo) X, 19

Physics Department, Manchester University (Group Photo) IV, 70

Rasmussen, Manne (Photo) IV, 20

Redpath, Grace (Photo) VI, 24

Redpath Museum. Museology students and staff (Group Photo) III, 87, Geological exhibit (Photo) 94

Redpath, Peter (Photo) VI, 25

Renaud, L.O. (Photo) III, 83

Richler, Mordecai (Photo) VI, 150

Ritchie, Grace (Photo) VIII, 16

Robertson, Harold Rocke (Photo) X, 26

Roosevelt, Eleanor (Photos) II, 9; IV, 152

Rutherford, Ernest I. (Photos) I, (4a); II, 113; V, 158; IX, 108; X, 10 
Ruttan, R. F. (Photo) IX, 106

Santa Cruz, Hernan (Photo) IV, 147

Scott, Frank R. (Charcoal Portrait) II, 99; (Photo) III, 7

Scott, W.B. (Photo) I, (79b)

Skriabin, Adelaida Mikhailovna (Photo) VIII, 149

Smith, Sir Donald (Portrait) VI, 93

Soddy, Frederick (Photos) IX, 108; X, 10

Steacie, E. W. R. (Photo) IX, 108

Stephenson, John (Portrait) VIII, 139

Strathcona, Lord (Portrait) VI, 93

Todd, Isabella McLennean McMeekin Dulaney (Photo) VIII, 43

Vaillancourt, Émile (Photo) VI, 122

Vaughan, Susan Cameron (Photo) V, 48

Wainwright, Arnold (Photos) I, (79b); V, 75

Workman, Thomas (Photo) IX, 106

\section{PLACES OR SCENES}

A.H. Scott House (Photo) IX, 52

Cape Desolation, Southwest Greenland (Photo) IV, 14

Esquimalt, Sailing Ship (Photo) VII, 142

Kuunatt Mountain, Southwest Greenland (Photo) IV, 17

Macdonald Chemistry Building (Photo) VIII, 67

Macdonald College. Main Building (Photos) VIII, 71; Dining Room, 71

Macdonald Engineering Building (Photo) VIII, 67; (Nobbs drawing) IX, 89

Macdonald Physics Building (Photos) VII, 156; VIII, 67

Macdonald, William. Residence. Prince of Wales Terrace. Living Room (Photo) VIII, 62

McCord, David Ross. Residence. Temple Grove, Montreal (Photo) III, 30

McGill College, 1852 IX, 21

McGill University (Nobbs colour print) IX, 83, (Nobbs drawing) 86

McGill University Library (Nobbs drawing) IX, 89

McGill University Union (Nobbs drawing) IX, 87

McLennan Library Building (Photo) VII, 153

Montreal Medical Institution (Drawing) VIII, 141

Montreal Veterinary College (Photo) X, 104 
Mount Atter, Greenland (Photo) IV, 30

Nobbs, Percy Erskine. Residence (Photo) IX, 90, Garden (Painting) 91

Notman Studio (Photo) VII, 133, (Lithograph) 134

Polish Institute Library (Photos) VI, 157; Reading room, 160

Redpath Hall. Organ (Photos) VI, 34; Stained glass windows, 40

Redpath Library (Photo, exterior) VI, 23, 27, (interior) 26; (Exterior, pen and ink drawing) 32; Reading Room, (Photos) 28, 35, 36, 38, 44; (Pen and ink drawing) 33; (Photos) Cataloguing Room, 29; Delivery Counter, 30; Exhibition Gallery, 37; Circulation Desk, 39; Book Stacks, 41; Cataloguers, 42, 43.

Redpath Museum. (Photos) I, (50a); (interior) VII, 57

Sarfartok River, Kugsuak, Greenland (Photos) IV, 24, 29

\section{OBJECTS}

Alexander III. Numismatic profile (Coin) IV, 117

Apulian Red-figure Bell Krater (4th century B.C.) IX, 151

Arethusa. Numismatic profile (Coin) IV, 117

Art Cabinet (Gift of Mrs. Elizabeth Lewis) VI, 163

Beament, T.H. H.M.C.S. Labrador (Painting), Stranded Iceberg (Painting) V, 85; Something in Sight

(Painting), Winter Travel Baffin Island (Painting) V, 86

Brymner, William Gathering Wild Strawberries (Painting) V, 87; Autumn River (Painting) V, 88

Canadian Medical Library Association Commemorative Medal of Charlton, Gould, Osler (Photo) II, 55

Carmichael, Franklin Whitefish Hills (Painting) V, 88

Carr, Emily B.C. Forest (Painting), Indian School, Lytton, B.C. (Painting) V, 89

Casson, A.J. Northern Lake (Painting), On the North Channel Little Current (Painting) V, 90

Coburn, F.S. Logging Team (Painting) V, 90

Coffin (Athropid) of Tjaoneferamen III, 116

Coins: Nero, Mint of Rome, A.D. $64-66$ (Photo) IV, 111; Galba of Padua, Mint of Rome (Photo), 114; Alexander III (Photo), 117, Head of Arethusa (Photo), 119

Condiment/Bonbon dish (Bronfman Estate) IX, 116

Dinner Dress (Fin-de-siécle, gift of Mme. Charles Taschereau) V, 226

Earthenware Jar. Pre-Dynastic-Early Dynastic Period. Koshtamna, Nubia III,116

Earthenware Platter (Davenport). View of Montreal III, 154

Edson, Allan Forest with Brook in Autumn (Painting), Forest with Shepherd and Sheep in Autumn (Painting) V, 92

Gagnon, Clarence Street in Dinan (Painting) V, 93

Galba of Padua. Numismatic profile (Coin: Mint of Rome) IV, 114 
Gordon, Hortense Country Store (Painting) V, 93

Greek Papyrus. Oxyrhynchus II, 66

Harris, Lawren Chestnut Tree, House, Barrie (Painting), Red Sleigh, House Winter (Painting) V, 94.

Hewton, Randolph St. Tite des Caps Charlevoix County (Painting) V, 95

Holgate, Edwin Woman Before a Window (Painting) V, 96

Holdstock, A. W. Creek Above LaPuce Falls, Quebec (Painting) V, 95

Holler, Wenceslaus Portrait of a Young Woman (Print) VI, 101

Hutchison, F.W. Autumn Landscape, Farm Near St. Urbain (Painting), Lake of Two Mountains in Winter (Painting), Stream with Geese, Charlevoix County (Painting) V, 97

Jackson, A.Y. Dawn in the Yukon (Painting), Great Bear Lake Near Port Radium (Painting) V, 98;

Autumn in Temagami (Painting) V, 99

Johnston, Franz Forest Interior (Painting) V, 100; Mountain Landscape (Painting) V, 101

Krieghoff, Cornelius Returning Home, Lake Memphramagog (Painting) V, 102; King's Walden (Painting) VII, 165; Plantation Near Guisborough, Yorkshire (Painting) VII, 166; Seignory of Ste-Anne de la Pérade (Painting) VII, 167; Sleepy Hollow (Painting) VII, 168

Leduc, Ozias Monsieur L'Abbé Vincent (Painting) V, 103; Still Life (Painting) V, 104

Lismer, Arthur Georgian Bay (Painting), Seal Cove, Grand Manan (Painting) V, 105; Cedar Swamp,

B.C. (Painting), Low Tide, Grand Manan (Painting) V, 106

Little, John Park Below Westmount Lookout (Painting) V, 107

Lock, Frederick Portrait of John Samuel McCord (Painting) III, 24, Portrait of Anne Ross McCord

(Painting) 27

Lyman, John Hamilton Harbour, Bermuda (Painting) V, 107

Macdonald, J.E.H. Mount Goodsir, Rockies (Painting) V, 108

Medal. Canadian Medical Library Association - Charlon, Gould, Osler (Photo) II, 55

Métis Wall Pocket. Beadwork, felt, cotton III, 152

Millard, C.S. Cascades, September (Painting) V, 108

Milne, David Spring Pool (Painting) V,109

Morris, Kathleen The Saddler's Shop, Ottawa (Painting) V, 110

Nero. Numismatic profile. (Coin: Mint of Rome, A.D. 64-66) IV, 111

Nobbs, Percy Macdonald Park (Painting) IX, 84-5

Papyrus, Greek. Oxyrhynchus II, 66

Pfeiffer, Gordon Ripening Grain, Saint Urbain (Painting), Fourth Range, Charlevoix County (Painting) V, 111; Cape Trinity (Painting), Laurentians, Winter (Painting) V, 112

Pilot, Robert Mount Murray from Pointe au Pic (Painting) V, 113; The Ramparts (Painting) V, 114; The Basilica at Twilight, Quebec City (Painting), Spring Day, St. Eustache (Painting) V, 115

Piranesi View of Ponte Fabrizio (Print), VII 159; View of the Temple of Sibyl (Print) VII, 161

Rembrandt The Descent from the Cross (Print) X, (34) 
Roberts, Goodridge Summer Montebello (Painting), Pine Tree on the Shore (Painting), Country Road on a Bright Day (Painting) V, 116; Reclining Nude (Painting), Still Life, Flowers and Fruit (Painting) V, 117; Phlox, Blue Cloth (Painting) V, 118

Robinson, Albert Corner on the St. Lawrence (Painting), Farm on the Hills, Knowlton (Painting) V, 119 Samovar (Bronfman Estate) IX, 114.

Serving Spoons/Forks. (Bronfman Estate) IX, 117

Stevens, Levi Portrait of Mary Todd Ferrier (Painting) IV, 180

Suzor-Coté, M.A. Spring Landscape Arthabasca (Painting), Eastern Townships (Painting) V, 120

Taylor, Fred. F.R. Scott. (Charcoal Portrait) II, 99

Tjaoneferamen. Anthropid Coffin III, 116

Universal Declaration of Human Rights (Poster) II, 11

Varley, F.H. Reflections, Garibaldi Park B.C. (Painting) V, 121

Verner, F.A. Buffalos on the Canadian Prairie (Painting) V, 121

Whale, Robert Indians with Dead Caribou and Bark Canoe at Big Rock, Memphramagog (Painting) V, 122 